\title{
Reducing medicines waste in the community
}

Jill Jesson Aston Business School, Aston University, Birmingham, UK, Rob Pocock M.E.L Research Ltd, Birmingham, UK and Keith Wilson School of Pharmacy, Aston University, Birmingham, UK

\begin{abstract}
There is increasing concern at the amount and cost of prescribed medicines that are unused or wasted and then have to be disposed of. Previous studies have used health promotion and Dispose Unwanted Medicines Properly campaigns targeted at the patient to describe and quantify the annual cost of waste. The reasons patients return unused drugs to pharmacies have also been explored. The paper focuses on patient explanations for not needing medication; categorized as: over-collection in the past, self-management strategies, changes in medical condition, other changes in patient circumstances, or the repeat medicines policy at the surgery. The aim of the original study was to make a measurable change in prescribed medicines with a reduction in medicines wastage, whilst at the same time achieving improved standards of pharmaceutical care. Information on patient needs and behaviour came from consultation in the pharmacy monitoring forms and interview. The study was based on two medical practices in the West Midlands, UK, comparing an outer city and an inner city population. The participants were general practitioners, pharmacists and 350 repeat prescription patients. Prescriptions were issued for two three-month periods. The outcome was that $23.8 \%$ of the prescribed items were not dispensed, at a value of $£ 13.1 \mathrm{~K}, 58 \%$ of the medications that would be expected to be regularly supplied were collected. The study suggests that closer professional management at the point of dispensing and an understanding of patient experiences can help reduce the amount of unwanted medication collected by patients.
\end{abstract}

Key words: community pharmacists; medicines waste; patients

\section{Introduction}

There is increasing recognition amongst health service funders (Department of Health, 2000) and prescribers (Royal College of Physicians, 1997) of the growing amount and cost of prescribed medicines that are unused or wasted. At the same time, there is increasing evidence in developed countries that pharmaceutically active compounds leech from land fill sites and from sewerage plants and can enter the groundwater (Heberer, 2002). The extent of unwanted medication has usually been measured by pharmacists and costed by examining returned waste in Dispose Unwanted Medicines Properly (DUMP) campaigns, which invite patients

Address for correspondence: Jill Jesson, Aston Business School, Aston University, Birmingham, B4 7ET, UK. Email: j.k.jesson@aston.ac.uk to return all unused medication to the pharmacy, or in Brown Bag reviews, where patients are invited to take all medication to a pharmacist for review (Anon, 1992; Cromarty and Downie, 2001). The General Pharmaceutical Services Bulletin for England and Wales provides DUMP data by primary care trust (PCT) and health authority (HA) for the year 2002/2003 (Department of Health, 2004). However, only 215 of the 342 PCTs in England and five HA in Wales are able to provide data.

The purpose of this paper is first to review the limited literature on medication waste and then to draw on data produced during a repeat dispensing pilot study which adds to our understanding of the patients' use of their medication (Wilson et al., 2002). This study was one of 12 pilots that were funded by the Department of Health through the Community Pharmacists Wider Role Initiative in 
April 1997 to explore the pharmacist's medicine management role. At the same time we demonstrate the potential for community pharmacists to tackle the problems of waste National Health Service (NHS) drugs resources.

\section{Waste and unwanted medicines}

In the UK, the National Pharmaceutical Association (NPA) estimated the annual value of wasted dispensed drugs at $£ 37.6$ million (Anon, 2000a) compared with a total expenditure on drugs in 2002 of $£ 6.8$ billion (Department of Health, 2003). Considerable publicity has been given to attempts to reduce this. For example, Wiltshire Health Authority mounted an education campaign after noting that almost $2 \%$ of its $£ 53$ million drug budget was being spent on medicines which are never used and blame was attributed to patients ordering multiple item repeat medications (Anon, 2000b). This is an international problem. In Sweden, researchers who collected returned drugs from 100 pharmacies for one year calculated a mean of 17.3 tonnes per month of waste, but noted that the unused medication was of relatively small cost items (Ekedahl et al., 2003). In the UK, the government funded Disposal of Old Pharmaceuticals (DOOP) scheme recorded a total collection of 609 tonnes from community pharmacies in the year ending March 2002 (Department of Health, 2002). However, these types of study only measure the medication returned to pharmacies, there are no data on medication returned to general practitioner (GP) surgeries. In addition, there is qualitative evidence that householders also rid themselves of superfluous medications by disposal through the municipal waste bin collection and by flushing to the public sewerage system (Chartered Institute of Wastes Management, 2000). A proper knowledge of this hidden behaviour and its environmental impact has yet to be developed and is not pursued further here.

\section{Explanations for waste}

Failure in the repeat prescribing process is a commonly held explanation for medicines waste. A conference for health professionals on wasteful prescribing was held in 2001 to discuss ways of tackling the problem (Bellingham, 2001). In the
UK, problems with the management of the repeat prescribing system are well documented (Davidson et al., 1998; McGavock et al., 1999). These range from the practical, where doctors are 'exasperated by an increasingly time consuming but otherwise dull task' (Drury, 1982) to clinical concerns about the scale of long-term medication without proper review (Harris and Dadja, 1996). Others assert that many long-term medication reviews were actually inadequate (Zermansky, 1996). Whatever the cause, the impact on the patient can be drug stockpiling and poor compliance (Audit Commission, 1994; Bond, 2000).

The reasons why patients accumulate unused medication are less well understood. One study in West Yorkshire measured routinely returned drugs to 33 pharmacies over one month and so derived a profile of the returned drugs in terms of both value and British National Formulary (BNF) classification (Hawksworth et al.,1996). This study categorized reasons for the return of unwanted medication to the pharmacy as: death $(42.1 \%)$, too much stock at home (23.4\%), medication no longer required because changed by prescriber $(25 \%)$ and medicines had passed expiry date $(5.0 \%)$. The data imply that $67 \%$ of waste is unavoidable. A later, but similar, study based on four pharmacies in Wales measured 10 weeks of returns and added three more explanatory categories: medication stopped by patient, adverse effect from drugs, error of prescription, order or supply (Braybrook et al., 1999). We contend that three of these seven reasons for waste are clearly avoidable (see Table 1).

The limitation of both these studies is that they tell us only what was returned and limited categorical reasons for the return. Neither study sheds much light on how or why patients acquired the medication in the first place.

\section{Intervention to reduce waste}

To date there have been three main approaches to the problem of medicines waste. The first is through patient education using health promotion leaflets, DUMP campaigns and Brown Bag reviews. For example, the UK Pharmaceutical Services Negotiating Committee (PSNC) reports 17 DUMP campaigns and drug waste management projects undertaken since 1994 (Pharmaceutical Negotiating Committee, 2003). The second is by attention to prescribing, which can be seen as part of the wholesale 
Table 1 Reason for returning wasted medication and the extent to which they are avoidable

\begin{tabular}{ll}
\hline Category & Scope for reduction \\
\hline Death & Unavoidable, amenable to closer monitoring \\
Medication no longer required because changed by prescriber & Unavoidable \\
Too much stock at home & Avoidable \\
Medicines had passed expiry date & Avoidable (depends on drug) \\
Medication stopped by patient & Unavoidable \\
Adverse effect from drugs & Unavoidable \\
Error of prescription, order or supply & Avoidable \\
\hline
\end{tabular}

Source: Hawksworth et al., 1996; Braybrook et al., 1999.

review of prescribing management that has been ongoing over the last 10 years. This has been driven through increasing scrutiny by health service managers with a primary focus upon achieving better value for money through cost-effective prescribing (Davidson et al., 1997; Staunton, 1997). An increasing number of studies detail the contribution of pharmacists working in primary care with GPs to improve prescribing practice and enhance patient care (Sykes et al., 1996; Davidson et al., 1997; Goldstein et al., 1997; Wood et al., 1997; Burtonwood et al., 1998; Dowell et al.,1998; Geoghan et al., 1998; Petty et al., 2002). The third is through engagement of patients in their health care. Compliance is a description of patient behaviour in which patients are expected to take medicines as instructed. The more recent approach of concordance involves a process in which patient and prescriber reach agreement on medication in the expectation that engagement of the patient in the decision will lead to improved adherence (Royal Pharmaceutical Society, 1997).

\section{Better repeat prescribing as a means to cut waste}

An impressive number of studies have detailed the outcome of medication reviews in the medical practice, which offer insight into ways of measuring and reducing the amount of unwanted medicines. Many of the early reviews concentrated on improving the standard of prescribing by examining prescribing analysis costs (PACT) data, acting on proprietary and generic substitution, switching expensive for cheaper alternatives and identifying ineffective drugs for the indication stated. Following this initial focus, prescribing support pharmacists have moved on to tackle other repeat prescribing issues, including waste. The three most common problems identified on GP notes and patient record systems were inappropriate directions, inconsistent quantities and drugs no longer required. It has been suggested that greater attention to reviewing medication ordered on a repeat basis could greatly reduce drug costs (Goldstein et al., 1997) and by implication waste. The success of these prescribing initiatives was recognized through government policy, funding and support for every PCT from the National Collaborative Medicines Management services programme. Key aims were to identify and address unmet pharmaceutical need, to help patients to get the best out of their medicines and develop approaches which improve service efficiency and reduce waste (Jackson et al., 2002). Moreover, the National Service Framework for Older People (NSFOP) specified that all patients over 75 should normally have an annual review of their medication (Department of Health, 2001).

In comparison, we know less about outcomes when review takes place in the community pharmacy, where the patient case notes and prescribing history is not necessarily available. The repeat dispensing pilot study contributes to this part of the debate.

\section{Methods}

\section{The repeat dispensing pilot study}

The policy context of the repeat dispensing pilot was the engagement of pharmacists in patientcentred care to achieve better health outcomes through improved prescribing coupled with a reduction in waste (Audit Commission, 1994). The 
Birmingham repeat dispensing pilot study allowed community pharmacists to dispense repeat medication without the need for the patient to visit the surgery. One objective was to make a measurable change in prescribed medicines with a calculated reduction in medicines wastage, whilst at the same time achieving improved standards of pharmaceutical care. The study was based in two medical practices and seven community pharmacies within the West Midlands conurbation. Neither practice had undertaken a systematic clinical medication review of patients on repeat prescriptions. A more detailed description of this pilot study has been reported elsewhere (Wilson et al., 2002).

\section{Sample selection}

Experience elsewhere showed that inviting patients to voluntarily attend a community pharmacy for a review of their medication was not well taken up (Nathan et al., 1999). To overcome this problem, the patient sample was drawn using a four-stage strategy. Initial identification of potential patients was by prescriptions issued by the two practices and presented at the study pharmacies. The pharmacists checked their records to confirm repeat status and then the names were screened by the medical practitioners for deletion of any patient deemed clinically unsuitable. This group was invited by post to participate in the study. From a total of 618 repeat patients who agreed to participate, a sub-sample of 350 was enrolled into the intervention, distributed across seven pharmacies. Thus the patients were volunteers, selected on the basis of repeat medication prescribed and dispensed by the participating organizations. The intention was to work with a typical cross-section of patients, not to select by specified clinical or therapeutic criteria.

\section{The study intervention}

Patients were entered for six months using prescriptions issued by the practices for all medication authorized for repeat prescribing by the supervising GP. Two local research ethics committees approved the project design and instruments. The intention was to allow dispensing pharmacists to take a greater part in working with patients in the management of their medication. There was flexibility in the pharmacist's ability to become involved in the choice as to whether a) it was appropriate to dispense an individual item, and b) what quantity was required. Participating pharmacists were remunerated on a patient per capita basis rather than the normal method which is based upon a fee for each item dispensed. The intention was to remove any disincentive not to supply and to provide some additional payment to recompense for the extra pharmaceutical care provided in the project. Monitoring systems included patientpharmacist monitoring forms to record activity with duplicate pads of referral forms to allow communication back to the GP, while GPs had forms to notify medication changes to the pharmacist. It is the monitoring forms which provide the evidence for the findings described in the following section. In addition to identifier detail, the pharmacy monitoring form provided information on six elements: the number of items not dispensed; whether there were any issues of interactions, side effects, adverse drug reactions (ADR), compliance or any other drug related concerns; details of any problems discussed; whether a referral was made; details of any supply issues; who collected the medication; consultation time.

\section{Results}

\section{Study sample}

The demographic profile of the selected subsample was: $62 \%$ female, $38 \%$ male; $89 \%$ white, $7 \%$ black Caribbean, $5 \%$ Asian. The age range was predominantly elderly, $8 \%$ under $42,25 \%$ were $43-62,67 \%$ over 62 . Eighty-eight per cent were exempt from payment, $5 \%$ had a prepayment certificate; $66 \%$ of participants had up to four repeat items, $14 \%$ six or more items. Seventy-five per cent had been on repeat medication for more than two years. The health professionals involved considered this to be a good cross-section of patients receiving repeat medication.

\section{Study findings: the prescription data}

At each ordering of medication, the pharmacists completed a patient monitoring form, entering details about the medication dispensed and free text on issues which arose during the consultation. A total of 167 free text entries were made. A total of 288 patients completed the full six months of 
the study and these patients had available to them a total of 1501 different medicines, each available for multiple supply. A total of 7153 supplies could have been made during the six months of the project, of which 5454 were actually dispensed. Therefore $23.8 \%$ of the potential supplies were not made representing an ingredient value of $£ 13.1 \mathrm{~K}$. A detailed analysis of the cost savings of the Birmingham pilot study have been reported elsewhere (Wilson and Jesson, 2003). This reflects potential waste if the project had not been running.

Each medicine was categorized as either essential for a chronic condition, which should be taken continuously, or as symptomatic relief to be taken in accordance with patient needs and therefore not necessarily continuously. We would expect medicines for chronic conditions to be collected regularly and medicines for symptomatic relief to be collected according to patient need. A total of $62 \%$ of the prescribed medicines were for chronic conditions. This means that $38 \%$ of the prescribed medicines would not necessarily be taken regularly and therefore if supplied regularly might generate waste. It is within this group that there is potential for saving, if patients are supported in their decision concerning supply (Wilson and Jesson, 2003).

The pharmacist recorded each consultation and this showed how spending time discussing medication needs with the patient helps them to make a better choice each month when they decide which medication to collect. These records also demonstrated the increased difficulties when medication is collected by a carer or patient representative since these intermediaries frequently did not have an informed appreciation of the medication required.

\section{Analysis}

The free comments were typed up, coded by pharmacy and patient number. Using grounded analysis the selected data were searched for common patterns by a process of highlighting. Then each set of highlighted responses were re-allocated into themes (Easterby-Smith et al., 2002). This process was undertaken independently by two researchers until consensus was reached on labelling five categories: over-collection in the past, selfmanagement strategies, changes in medical condition, other changes in patient circumstances, repeat medicines policy administration at the surgery. The features of each category are summarized below, with examples to support the analysis.

- Over-collection in the past: This selection of comments made by the pharmacist at the point of supply shows that patients did need some regular help in ordering and managing the collection of their medication. The drugs referred to by the pharmacist are mainly analgesics, but others were involved.

First collection. Apparently had plenty left at home over past three months.

Plenty of Co-dydramol left from last time so not dispensed. Patient checked back and also has plenty frusemide left so again not dispensed.

Patient was going to order Dihydrocodeine but on talking to her she discovered she had plenty left.

- Self-management: Some patients were trying to manage their own care by adapting their medication to suit their own illness patterns, lifestyle and health needs. With help from the pharmacist in managing their medicines, that particular form of self-care can be supported and encouraged, but monitored in a friendly but informative way. Since the medical practices did not monitor usage of medicines, this support by a pharmacist was an additional service not currently provided.

Only take half a tablet of the $1 \mathrm{mg}$ Warfarin, so only 14 dispensed.

Doesn't take full quantity of co-codamol so gave half.

- Changes in medical condition: It is inevitable that in patients with chronic conditions their medication profile will change over time, sometimes changes are made from secondary care, as the following comments illustrate.

Hands have cleared up since stopped work, so mexosyn and co-proxamol rarely used now as not required.

No angina attacks recently - still got plenty of unopened GTN tabs.

Patient has been in hospital so has not used her last medication, barring the paracetamol - so nothing else dispensed. 
- Repeat medicines policy administration at surgery end: This fifth category covers the medicines management procedures at the surgery where effective repeat medication management systems should pick up items no longer prescribed or needed.

\section{No longer taking one set of medication.} Taken off by GP but still on script.

The study identified a number of examples where medicine management discussions with patients in the pharmacy lead to more cost-effective use of NHS resources. Three-quarters of patients collected their own medication, supporting other study findings. However, the $25 \%$ who collected for another emerged as the group where the likelihood for confusion over quantities of medication needed was more pronounced and therefore as the group who probably need most support and time investment. The comments written by pharmacists illustrate the need for direct access to the patient, whether by domiciliary visit or by telephone. The following examples of discussion between pharmacists and patient representatives illustrates the type of issues that can arise when the patient is not present.

Son came to collect medication - did not know which items were required, therefore I phoned the patient at home.

Patient was using nitrolingual spray up to 10 times daily according to daughter, even though not experiencing any pains in chest. Have filled in GP referral form.

Sends a representative who doesn't know what patient needs. Just says give everything sent him back to ask patient what he really needs.

\section{Discussion}

This six-month pilot study allowed dispensing pharmacists to check and document the collection and use of medications on repeat prescriptions and gave us insight into the problem of accumulating unused medicine. In quantitative terms we have shown that just under a quarter of the prescribed items were not collected and this indicates the potential for a repeat dispensing service to reduce medication waste. However, the study also shows that the process of repeat medication supply is complex. The patients in our study had available to them an average of over five medications each month and these included those for chronic ongoing conditions where continuous, regular therapy is essential and those for variable conditions that the patient has to manage according to symptoms.

Our study adds to the debate on waste medicines, by providing more than quantitative data on waste, we demonstrate that a better professional understanding and involvement at each point of supply can benefit individual patients care and at the same time reduce the potential for unwanted supply. This finding has been recognized in the design of the PCT Pathfinder Repeat Dispensing project which commenced in 2003. Repeat Dispensing Services can also complement other PCT requirements of the NSFOP and the Medicines Management Collaborative reviews, which are undertaken periodically at the point of prescribing.

The limitations of the study are the size and scope of a pilot study. Nonetheless the quantitative data on nondispensed medication is similar to findings from other repeat dispensing studies (Wilson and Jesson, 2003) and therefore fairly robust. It was the additional qualitative data recorded by pharmacists that offered us a chance to explore, using a grounded inductive approach, the reasons that patients gave for not needing their next prescription dispensed. By comparison, other studies on returned waste used precoded categories to record reasons for returning unused medication.

Debates about the amount and cost of unused medication returned for disposal summarized in the literature review above, have caused considerable concern at the waste of resources, cost to the NHS and the cost of disposal and potential damage to the environment. Interventions to date have focused on four approaches: publicity to cut down on waste through Brown Bag reviews, health promotion leaflets, DUMP campaigns and attempts to achieve better compliance (concordance). Many studies have shown that it is possible to achieve change through clinical review. The changes can be made periodically at the point of prescribing at the surgery or regularly at the point of supply at the pharmacy. This study was based at the final point in the supply cycle, that of dispensing when there 
is the potential for direct contact between the patient and a health professional. As our data show, there can be a change in circumstances which may not automatically be picked up at the issue of repeat medication prescription.

The findings from this study emphasize the complexity of the decision process on medication needs, which requires knowledge of both the function of the medication and the underlying medical condition. Even during this pilot there was a mismatch between regularity of supply and the nature of the medication and there were a number of medications for chronic conditions that were not regularly supplied. However, it is important to recognize that the decision as to whether a medication was needed lay with the patient or their representative. Our study reinforces the need for patient guidance in this decision process and therefore the potential for professional support.

In the past there has been an emphasis on measuring waste by volume, and for developing a theory about the causes of waste that had a tendency to blame the patient through assumption of noncompliance with prescribing directions. Theoretically an approach to prescribing based upon 'concordance' might be expected to reduce medicines waste (Royal Pharmaceutical Society, 1997), however, the evidence from earlier studies (Table 1) shows that many of the reasons given by patients for returning unwanted drugs are unavoidable, such as 'death of the patient', 'no longer required, changed by prescriber' and 'stopped by a patient experiencing ADR'. Others are avoidable and these include patient related issues such as 'too much stored', passed expiry date and problems relating to the prescribing process such as errors in prescription ordering. The analysis of qualitative comments from the study confirm that many instances are avoidable: we have added some of the contextual background to demonstrate reasons, for example, why 'too much is stored'.

There has been increasing recognition of the importance of the prescribing process but this is inevitably remote from the patient with only periodic review between prescriber and patient. In this study, we have focussed upon the point of supply within the community pharmacy. By drawing on the pharmacist's comments at the point of supply we have greater insight into the supply side and patient behaviour. We believe that this indicates a need for better professional support. Optimizing supply must be a partnership process that involves prescriber, supplier and patient and the repeat dispensing process that we have described embraces all three elements. It provides a method of waste minimization, but more importantly, a support process for patient decisions on long-term medication needs.

\section{References}

Anon. 1992: Multiple medicines in the bag. Lancet 339, 1475.

Anon. 2000a: Huge waste of medicines claimed. Pharmaceutical Journal 264, 228.

Anon. 2000b: Wiltshire Health Authority highlights medicines waste. Pharmaceutical Journal 264, 426.

Audit Commission. 1994: A prescription for improvement. Towards more rational prescribing in general practice. London: Her Majesty's Stationery Office.

Bellingham, C. 2001: How pharmacists can help to prevent wastage of prescribed medicines. Pharmaceutical Journal 267, 741-42.

Bond, C. 2000: Evidence based pharmacy. London: Pharmaceutical Press.

Braybrook, S., John, D.N. and Leong, K. 1999: A survey of why medicines are returned to pharmacies. Pharmaceutical Journal 263, R30.

Burtonwood, A., Hinchliffe, A. and Tinkler, G. 1998: A prescription for quality: a role for the clinical pharmacist in general practice. Pharmaceutical Journal 261, 678-80.

Chartered Institute of Wastes Management. 2000: Healthcare waste management and minimisation, a guidance document. Northampton: Chartered Institute of Wastes Management (CIWM).

Cromarty, E. and Downie, G. 2001: Drug wastage - what is acceptable? Pharmaceutical Journal 267, 424.

Davidson, W., Collett, J.H., Jackson, C. and Rees, J.A. 1998: An analysis of the quality and cost of repeat prescriptions. Pharmaceutical Journal 260, 458-60.

Davidson, W., Collett, J.H., Jackson, C. and Rees, J.A. 1997: Identification and costs of prescriber defined repeat prescribing. International Journal of Pharmacy Practice 5, 46-49.

Department of Health. 2000: Pharmacy in the future - implementing the NHS plan. London: Department of Health.

Department of Health. 2001: Medicines and older people. Implementing medicine related aspects of the NSF for older people. London: Department of Health.

Department of Health. 2002: General pharmaceutical services in England and Wales 1992/3 to 2001/2. London: Department of Health.

Department of Health. 2003: Prescriptions dispensed in the community. Statistics for 1992 to 2002: England. London: Department of Health. 
Dowell, J., Cruikshank, J., Bain, J. and Staines, H. 1998: Repeat dispensing by community pharmacists: advantages for patients and practitioners. British Journal of General Practice 48, 1858-59.

Drury, V.W.M. 1982: Repeat dispensing - a review. Journal of Royal College of General Practitioners 32, 42-45.

Ekedahl, A., Wergman, L. and Rydberg, T. 2003: Unused drugs in Sweden measured by returns to pharmacies. Journal of Social and Administrative Pharmacy 20, 26-31.

Geoghan, M., Pilling, M., Holden, J. and Wolfson, D. 1998: A controlled evaluation of the effect of community pharmacies on general practice prescribing. Pharmaceutical Journal 261, 864-66.

Goldstein, R., Hulme, H. and Willits, J. 1997: Reviewing repeat prescribing - general practitioners and community pharmacists working together. International Journal of Pharmacy Practice 6.

Harris, C.M. and Dadja, R. 1996: The scale of repeat dispensing. British Journal of General Practice 46, 649-53.

Hawksworth, G.M., Wright, D.J. and Chrystyn, H. 1996: A detailed analysis of the day to day unwanted medicinal products returned to community pharmacies for disposal. Journal of Social and Administrative Pharmacy 13, 215-22.

Heberer, T. 2002: Occurrence, fate and removal of pharmaceutical residues in the aquatic environment: a review of recent research data. Toxicology Letters 131, 5-17.

Jackson, C., Seal, R., Queenborough, R., Cantrill, J. and Devlin, M. 2002: Modernising medicines management. A guide to achieving benefits for patients, professionals and the NHS. Liverpool: National Prescribing Centre (NPC) and the National Primary Care Research and Development Centre (NPCRDC).

McGavock, H., Wilson Davis, K. and Connolly, J.P. 1999: Repeat prescribing management - a cause for concern. British Journal of General Practice 49, 343-47.
Nathan, A., Goodyer, L. and Lovejoy, A. 1999. Brown bag medication reviews as a means of optimising patients use of medications and of identifying potential clinical problems. Family Practice 16, 278-82.

Petty, D.R., Zermansky, A.G., Raynor, D.K., Lowe, C.J., Freemantle, N. and Vail, N. 2002:A clinical medication review by a pharmacist of elderly patients on repeat medications in general practice - pharmacist interventions and review outcomes. International Journal of Pharmacy Practice 10,39-45.

Pharmaceutical Negotiating Committee. 2003: Community Pharmacy Services Database. http://psncdatabase.co.uk/result.php (retrieved 18 February 2005). London: PSNC.

Royal College of Physicians. 1997: Medication for older people. London: Royal College of Physicians.

Royal Pharmaceutical Society of Great Britain. 1997: From compliance to concordance. London: Royal Pharmaceutical Society of Great Britain (RPSGB) and Merck Sharpe and Dohme.

Staunton, N. 1997: Pharmacy drug interaction and cost saving intervention study. A report to the Department of Health. Hampshire: Isle of Wight Health Authority.

Sykes, D., Westwood, P. and Gilleghan, J. 1996: Development of a review programme for repeat prescription medicines. Pharmaceutical Journal 256, 458-60.

Wilson, K.A. and Jesson, J.K. 2003: Dispensing activity in a community pharmacy-based repeat dispensing pilot project. International Journal of Pharmacy Practice 11, 225-32.

Wilson, K.A., Jesson, J.K., Varnish, J., Pocock, R. and Barton, A. 2002: The Birmingham community pharmacy repeat dispensing project. Pharmaceutical Journal 269, 20-24.

Wood, K.M.G., Mucklow, J. and Boath, E. 1997: Influencing prescribers in primary care: a collaboration between clinical pharmacology and clinical pharmacy. International Journal of Pharmacy Practice 5, 1-5.

Zermansky, A.G. 1996: Who controls repeats? British Journal of General Practice 46, 643-47. 